\title{
Statistical Inference For K Exponential Populations Under Joint Progressive Type-I Censored Scheme
}

\author{
O.E. Abo-Kasem ${ }^{1}$ and Mazen Nassar ${ }^{1,2}$ \\ ${ }^{1}$ Department of Statistics, Faculty of Commerce, Zagazig University, Egypt \\ ${ }^{2}$ Department of Statistics, Faculty of Science, King Abdulaziz University, Kingdom of Saudia \\ Arabia
}

\begin{abstract}
In this article, the maximum likelihood estimators of the $\mathrm{k}$ independent exponential populations parameters are obtained based on joint progressive typeI censored (JPC-I) scheme. The Bayes estimators are also obtained by considering three different loss functions. The approximate confidence, two Bootstrap confidence and the Bayes credible intervals for the unknown parameters are discussed. A simulated and real data sets are analyzed to illustrate the theoretical results.
\end{abstract}

Keywords: Joint progressive Type-I censored scheme; Maximum likelihood estimation; Confidence bounds; Bootstrap intervals; Bayesian estimation; Squared-error loss; LINEX loss; general entropy loss. 


$$
\begin{aligned}
& \text { Notations } \\
& \text { pdf Probability density function } \\
& \text { cdf Cumulative density function } \\
& \text { iid Independent and identically distributed } \\
& \delta \quad \text { Number of censored stage } \\
& T_{j} \quad \text { Prefixed censoring times, } 1 \leq j \leq \delta \\
& \bar{F}_{\square}\left(T_{j}\right) \quad 1-F_{\square}\left(T_{j}\right) \text {, Survival functions of the } h \text { populations at } T_{j} \text {. } \\
& n_{1}, n_{2}, \ldots, n_{\square} \quad \text { Samples of sizes } \\
& N \quad \sum_{\square=1}^{k} n_{\square} \text {, The total sample size } \\
& W=\left(w_{1}, w_{1}, \ldots, w_{N}\right) \quad \text { The order statistics of the } N \text { random variables } \\
& r_{j} \quad \text { Number of units failed in } T_{j-1}, T_{j} \\
& s_{j}(\square) \quad \text { Number of units withdrawn at } T_{j} \text { belonging to the } h^{\text {th }} \\
& \text { samples } \\
& R_{j} \quad \sum_{\square=1}^{k} S_{j}(\square) \text {, Number of units censored at time } T_{j} \\
& =\left(z_{1}(\square), z_{2}(\square), \ldots, z_{\delta}(\square) \text {. Define as } z_{i}(\square)=\left\{\begin{array}{l}
1, \text { if } \square=\square_{i} \\
0 \text { ot } \square \text { erwise }
\end{array}\right.\right. \\
& M_{r}(\square) \quad \sum_{i=1}^{r} z_{i}(\square) \text {, Denote the number of } X_{\square}-\text { failures in } W \text { for } \\
& 1 \leq \square \leq k \\
& r \quad \sum_{\square=1}^{k} M_{r}(\square) \text {, The total number of complete failures } \\
& L \quad \text { Likelihood function } \\
& \text { I(.) Fisher's information matrix } \\
& \pi_{\square}\left(\lambda_{\square}\right) \quad \text { Prior density for the parameters } \lambda \\
& \pi\left(\lambda_{1}, \lambda_{2}, \ldots, \lambda_{k} \backslash \text { data }\right) \quad \text { Posterior density for the parameters } \lambda_{\square} \\
& \hat{\lambda} \\
& \hat{\lambda}_{\square B S} \\
& \hat{\lambda}_{\square B L} \\
& \hat{\lambda}_{\square B E} \\
& \text { Maximum likelihood estimators of } \lambda_{\square} \\
& \text { Bayes estimators of } \lambda_{\square} \text { under the SE loss function } \\
& \text { Bayes estimators of } \lambda_{\square} \text { under the LINEX loss function } \\
& \text { Bayes estimators of } \lambda_{\square} \text { under the GE loss function }
\end{aligned}
$$




\section{Introduction}

The joint censoring scheme is a very common way in conducting comparative life-tests of products from various units within the same facility. Suppose products are being produced by $\mathrm{k}$ different lines under the same conditions, and that $\mathrm{k}$ independent samples of sizes $n_{\square}, 1 \leq$ $\square \leq k$ are selected from these $\mathrm{k}$ lines and placed simultaneously on a life-testing experiment. Then, in order to reduce the cost and the experimental time, the experimenter may choose to terminate the life-testing experiment before complete information on failure times for all experimental units. Data arises from these experiments are called joint censored data. In this situation, the experimenter may be interested in either point or interval estimation of the mean lifetimes of units produced by the different $\mathrm{k}$ lines. In the literature, there were four types of joint schemes, namely, joint Type-II, joint progressive Type-II, joint progressive Type-I and joint type-I progressive hybrid censoring schemes. Balakrishnan and Rasouli (2008) developed the likelihood inference for the parameters of two exponential populations under joint Type-II censoring. Shafay et al. (2013), Ashour and Abo-Kasem (2014 a,b,c), considered the jointly Type-II censored sample. Rasouli and Balakrishnan (2010) studied the statistical inference of two exponential populations under the joint progressive Type-II censoring. Parsi et al. (2011) and Doostparast et al. (2013) considered the jointly progressive type-II censored sample.

Balakrishnan and Feng (2015) generalized the work of Balakrishnan and Rasouli (2008) by considering a jointly Type-II censored sample arising from $\mathrm{k}$ independent exponential populations. Also, Balakrishnan et al. (2015) generalized Rasouli and Balakrishnan (2010) work by considerring a jointly progressive Type-II censored sample arising from $\mathrm{k}$ independent exponential populations. Abo-Kasem et al. (2019) proposed a joint type-I progressive hybrid censoring scheme and investigated the estimation problems in the case of exponential distribution. Recently, Ashour and Abo-Kasem (2017) introduced JPC-I scheme and as a special case, joint Type-I censored scheme. They considered statistical inference for two exponential populations under both JPC-I and joint Type-I censored schemes. Abo-Kasem and Nassar (2019) developed the estimation problems of two Weibull populations with the same shape parameter under JPC-I censoring scheme. They obtained the maximum likelihood estimators (MLEs) and the approximate confidence intervals. They also obtained the Bayes estimates using squared error and LINEX loss functions under the assumption of independent gamma priors.

The JPC-I scheme in comparison to other joint censoring schemes provides an important advantage of the known termination time point of the life-testing experiment. From the experimenter point of view, this makes the JPC-I scheme very appealing for its implementation in practice. In spite of such a practical advantage, most of the inferential studies carried out in the literature focused on joint Type-II and joint progressive Type-II censoring schemes. For this reason, our aim in this paper is to investigate the point and interval estimation of $\mathrm{k}$ independent exponential populations under the JPC-I scheme. The rest of the paper is organized as follows: we formulate the problem in Section 2. The maximum likelihood estimation for $\mathrm{k}$ exponential populations and the approximate confidence intervals are obtained in Section 3. Section 4 describes the various bootstrap confidence intervals. In Section 5, we obtain the Bayes estimators under squared error, LINEX and general entropy loss functions as well as the Bayesian credible intervals for the parameters. In Section 6, a simulated and real data sets are analyzed. The paper is concluded in Section 7. 


\section{Model Description and Notation}

Suppose that $\left(X_{1}, \ldots, X_{N}\right)$ are $\mathrm{N}$ jointly distributed random variables, with $\left\{X_{1}, \ldots, X_{N}\right\}=$ $\left\{X_{11}, \ldots, X_{1 n_{1}} ; X_{21}, \ldots, X_{2 n_{2}} ; X_{k 1}, \ldots, X_{k n_{k}}\right\}$, and $N=\sum_{h=1}^{k} n_{h}$. Suppose $X_{11}, X_{12}, \ldots, X_{1 n_{1}}$ are the lifetimes of $n_{1}$ specimens from production line $A_{1}$, and are independent and identically distributed (iid) variables from a population with cumulative distribution function (cdf) $F_{1}(x)$ and probability density function (pdf) $f_{1}(x)$. Similarly, $X_{21}, X_{22}, \ldots, X_{2 n_{2}}$ are the lifetimes of $n_{2}$ specimens from production line $A_{2}$, and are assumed to be a sample from pdf $f_{2}(x)$ and cdf $F_{2}(x)$, and so on, with $X_{k 1}, X_{k 1}, \ldots, X_{k n_{k}}$ denoting the lifetimes of $n_{k}$ specimens from production line $A_{k}$ being iid variables from pdf $f_{k}(x)$ and $\operatorname{cdf} F_{k}(x)$. Denote the order statistics of these $\mathrm{k}$ random samples by $W_{1} \leq W_{2} \leq \ldots \leq W_{N}$, where $\mathrm{N}$ is the total sample size. Now, a JPC-I scheme between the k samples is implemented as follows: at the time $T_{1}, R_{1}$ units are randomly withdrawn from the remaining $N-r_{1}\left(r_{1}\right.$ number of units failed in time interval $\left.\left(T_{0}-T_{1}\right)\right)$ surviving units. Next, at the second time $T_{2}, R_{2}$ units are randomly withdrawn from the remaining $N-R_{1}-r_{1}-r_{2}\left(r_{2}\right.$ number of units failed in time interval $\left(T_{1}-T_{2}\right)$ ) surviving units, and so on. Finally, at the time $T_{\delta}$ all remaining $R_{\delta}=N-\sum_{j=1}^{\delta} r_{j}-\sum_{j=1}^{\delta-1} R_{j}$ ( $r_{\delta}$ number of units failed in time interval $\left(T_{\delta-1}-T_{\delta}\right)$ ), surviving units are withdrawn from the life-testing experiment, the total number of complete failures $r=\sum_{j=1}^{\delta} r_{j}$ as well as the progressive censoring scheme $\left(R_{1}, \ldots, R_{\delta}\right)$ are prefixed and has the decomposition $R_{j}=$ $\sum_{h=1}^{k} s_{j}(h), 1 \leq j \leq \delta$, where $s_{j}(h)$ is the number of units withdrawn at the $\delta$ fixed times $T_{1}, T_{2}, \ldots, T_{\delta}$ belonging to the hth sample and these are unknown and are latent random variables. The data observed in this form will consist of $(Z, R, W)$, where $W=$ $\left(w_{1}, w_{1}, \ldots, w_{r}\right), \quad w_{i} \in\left\{X_{\square_{i} 1}, X_{\square_{i} 2}, \ldots, X_{\square_{i} n_{i}}\right\}$ for $1 \leq h_{1}, h_{2}, \ldots, h_{\delta} \leq k$. Moreover, associated to $\left(h_{1}, h_{2}, \ldots, h_{\delta}\right)$, let us define $z=\left(z_{1}(\square), z_{2}(\square), \ldots, z_{\delta}(\square)\right)$ as

$$
z_{i}(\square)=\left\{\begin{array}{l}
1, \text { if } \square=\square_{i} \\
0 \text { ot } \square \text { erwise }
\end{array} .\right.
$$

Finally, let $M_{r}(h)=\sum_{i=1}^{r} z_{i}(h)$ denote the number of $X_{h}$ - failures in W for $1 \leq h \leq$ $k$ and $r=\sum_{h=1}^{k} M_{r}(h)$. Then the likelihood of $(Z, R, W)$ is given as

$$
L=C \prod_{i=1}^{r} \prod_{\square=1}^{k}\left(f_{\square}\left(w_{i}\right)\right)^{z_{i}(\square)} \prod_{j=1}^{\delta} \prod_{\square=1}^{k}\left(\bar{F}_{\square}\left(T_{j}\right)\right)^{s_{j}(\square)},
$$

where $\mathrm{C}$ is a constant and $\bar{F}_{h}\left(T_{j}\right)=1-F_{h}\left(T_{j}\right)$ is the survival function of the $\mathrm{h}$ populations. Note that, the special case of joint Type-I censoring scheme is obtained when we set $R_{1}=$ $R_{1}=\ldots=R_{\delta-1}=0$ so that $R_{\delta}=N-r$ in which case we will have $s_{j}(h)=0$ and $s_{\delta}(\square)=$ $n_{\square}-M_{r}(\square)$ for all $1 \leq \square \leq k$, where $M_{r}(\square)$ are completely observed failures from $\mathrm{h}$ samples.

\section{Maximum Likelihood Estimators and Inference}

Suppose that the k populations are exponential with pdf and cdf, respectively, as $f_{\square}(x)=$ $\lambda_{\square} e^{-\lambda \square x}$ and $F_{\square}(x)=1-e^{-\lambda \triangleright x}, \lambda_{\square}>0, x>0$, for $1 \leq \square \leq k$. In this case, the likelihood function in (1) becomes 


$$
\begin{aligned}
& L=C \prod_{\square=1}^{k} \lambda_{\square}^{M_{r}(\square)} \prod_{i=1}^{r} \prod_{\square=1}^{k}\left(e^{-\lambda_{\square} w_{i}}\right)^{z_{i}(\square)} \prod_{j=1}^{\delta} \prod_{\square=1}^{k}\left(e^{-\lambda_{\square} T_{j}}\right)^{s_{j}(\square)} \\
= & C \prod_{\square=1}^{k} \exp \left\{M_{r}(\square) \ln \left(\lambda_{\square}\right)-\lambda_{\square}\left(\sum_{i=1}^{r} z_{i}(\square) w_{i}+\sum_{j=1}^{\delta} s_{j}(\square) T_{j}\right)\right\} .
\end{aligned}
$$

From (2), we can obtain the MLE of $\lambda_{\square}$ for $1 \leq \square \leq k$, as

$$
\hat{\lambda}_{\square}=\frac{M_{r}(\square)}{\sum_{i=1}^{r} z_{i}(\square) w_{i}+\sum_{j=1}^{\delta} s_{j}(\square) T_{j}}
$$

To obtain the approximate confidence intervals of the unknown parameters, we first obtain the elements of the information matrix. Let $I\left(\lambda_{1}, \lambda_{2}, \ldots, \lambda_{k}\right)=$ $\left(I_{i, q}\left(\lambda_{1}, \lambda_{2}, \ldots, \lambda_{k}\right)\right), i, q=1,2, \ldots, k$, denote the Fisher information matrix of the parameters $\left(\lambda_{1}, \lambda_{2}, \ldots, \lambda_{k}\right)$, where $I_{i, q}\left(\lambda_{1}, \lambda_{2}, \ldots, \lambda_{k}\right)=-E\left(\frac{\partial^{2} \ln L}{\partial \lambda_{i} \partial \lambda_{q}}\right)$. From (2), we have $I_{i, q}\left(\lambda_{1}, \lambda_{2}, \ldots, \lambda_{k}\right)=0$ if $i \neq q$. Consequently, the observed Fisher information matrix is given by

$$
I\left(\hat{\lambda}_{1}, \hat{\lambda}_{2}, \ldots, \hat{\lambda}_{k}\right)=-\operatorname{Diag}\left(\left.\frac{\partial^{2} \ln L}{\partial \lambda_{1}^{2}}\right|_{\lambda_{1}=\widehat{\lambda}_{1}},\left.\frac{\partial^{2} \ln L}{\partial \lambda_{2}^{2}}\right|_{\lambda_{2}=\widehat{\lambda}_{2}}, \ldots,\left.\frac{\partial^{2} \ln L}{\partial \lambda_{k}^{2}}\right|_{\lambda_{k}=\widehat{\lambda}_{k}}\right)
$$

where

$$
\left.\frac{\partial^{2} \ln L}{\partial \lambda_{\square}^{2}}\right|_{\lambda_{\square}=\widehat{\lambda}_{\square}}=-\frac{M_{r}(\square)}{\lambda_{\square}^{2}}
$$

Based on the asymptotic normality of the MLEs, the approximate $100(1-\alpha) \%$ confidence intervals of $\lambda_{\square}$ is given by

$$
\hat{\lambda}_{\square} \pm Z_{\frac{\alpha}{2}} \sqrt{\frac{\hat{\lambda}_{\square}^{2}}{M_{r}(\square)}},
$$

where $Z_{\frac{\alpha}{2}}$ is the upper $\frac{\alpha}{2}$ percentage point of the standard normal distribution.

\section{Bootstrap Confidence Intervals}

In this section, two bootstrap confidence intervals are discussed. The two bootstrap methods are the percentile bootstrap (Boot-p) proposed by Efron (1982), and the bootstrap-t method (Boot-t) proposed by Hall (1988). We can use the following algorithm to obtain the Boot-p and Boot-t intervals,.

\section{a) Bootstrap Percentile Interval Procedure (Boot-p)}

(1) Compute the MLE $\hat{\lambda}_{h}$ of $\lambda_{h}$ based on JPC-I sample $(w, z, s)$.

(2) Use $\hat{\lambda}_{h}$ to generate a bootstrap JPC-I sample $\left(w^{*}, z^{*}, s^{*}\right)$, and compute the bootstrap estimate of $\lambda_{h}$, say $\hat{\lambda}_{\square}^{*}$, based on this bootstrap sample.

(3) Repeat step 2 B times to have $\hat{\lambda}_{\square}^{*(1)}, \hat{\lambda}_{\square}^{*(2)}, \ldots, \hat{\lambda}_{\square}^{*(B)}$.

(4) Arrange $\hat{\lambda}_{h}^{*(1)}, \hat{\lambda}_{h}^{*(2)}, \ldots, \hat{\lambda}_{h}^{*(B)}$ in ascending order to obtain $\hat{\lambda}_{\square}^{*[1]}, \hat{\lambda}_{\square}^{*[2]}, \ldots, \hat{\lambda}_{\square}^{*[B]}$.

(5) A two-sided $100(1-\alpha) \%$ Boot-p confidence interval for $\lambda_{h}$, say $\left[\hat{\lambda}_{\square L}^{*}, \hat{\lambda}_{\square U}^{*}\right]$ is given by

$$
\left(\hat{\lambda}_{\square L}^{*}, \hat{\lambda}_{\square U}^{*}\right)=\left(\hat{\lambda}_{\square}^{*\left(\left[B \frac{\alpha}{2}\right]\right)}, \hat{\lambda}_{\square}^{*\left(\left[B\left(1-\frac{\alpha}{2}\right]\right)\right.}\right), \square=1,2, \ldots, k
$$




\section{b) Studentized-t Interval Procedure (Boot-t)}

The Boot-t confidence intervals estimators are obtained according to the following steps:

(1-2) Same as the steps $1-2$ in (a).

(3) Compute the $\mathrm{t}$-statistic $T_{\widehat{\lambda}_{h}^{*}}=\frac{\left(\widehat{\lambda}_{h}^{*}-\widehat{\lambda}_{h}\right)}{\hat{S}_{\widehat{\lambda}_{h}^{*}}}$ where $\hat{S}_{\widehat{\lambda}_{\square}^{*}}$ is the bootstrap variance.

(4) Repeat steps 2-3 B times and obtain $T_{\widehat{\lambda}_{\square}^{*}}^{(1)}, T_{\bar{\lambda}_{\square}^{*}}^{(2)}, \ldots, T_{\bar{\lambda}_{\square}^{*}}^{(B)}$.

(5) Arrange $T_{\widehat{\lambda}_{h}^{*}}^{(1)}, T_{\widehat{\lambda}_{h}^{*}}^{(2)}, \ldots, T_{\widehat{\lambda}_{h}^{*}}^{(B)}$ in ascending order to obtain $T_{\hat{\lambda}_{\square}^{*}}^{[1]}, T_{\widehat{\lambda}_{\square}^{*}}^{[2]}, \ldots, T_{\widehat{\lambda}_{\square}^{*}}^{[B]}$.

(6) A two-sided $100(1-\alpha) \%$ Boot-t confidence interval for $\lambda_{h}$ say $\left[\hat{\lambda}_{\square, t L}^{*}, \hat{\lambda}_{\square, t U}^{*}\right]$, is given by

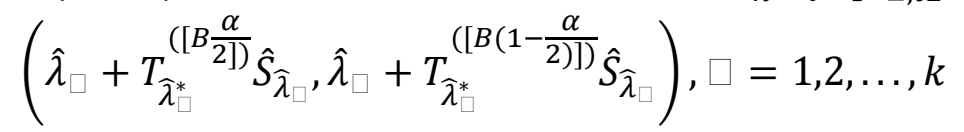

\section{Bayesian Inference}

Based on the likelihood function and the independent gamma prior distributions, viz. $G\left(a_{h}, b_{h}\right)$ for $1 \leq \square \leq k$, with pdf given by

$$
\pi_{\square}\left(\lambda_{\square}\right)=\frac{b_{\square}^{a_{\square}}}{\Gamma\left(a_{\square}\right)} \lambda_{\square}^{a_{\square}-1} e^{-\lambda b_{\square}},
$$

where $\Gamma($.$) denotes the complete gamma function. Combining (2) and (4), the joint posterior$ density of $\lambda_{1}, \lambda_{2}, \ldots, \lambda_{k}$ given the data is

$$
\pi\left(\lambda_{1}, \lambda_{2}, \ldots, \lambda_{k} \backslash \text { data }\right)=A \prod_{\square=1}^{k} \lambda_{\square}^{a_{\square}+M_{r}(\square)-1} \exp \left\{-\lambda_{\square}\left(b_{\square}+U_{\square}\right)\right\},
$$

where $A=\prod_{h=1}^{k} \frac{\left(b_{h}+U_{h}\right)^{a_{h}+M_{r}(h)}}{\Gamma\left(a_{h}+M_{r}(h)\right)}$ and $U_{\square}=\sum_{i=1}^{r} z_{i}(\square) w_{i}+\sum_{j=1}^{\delta} s_{j}(\square) T_{j}$.

From (5) the joint posterior density function of $\lambda_{1}, \lambda_{2}, \ldots, \lambda_{k}$ is a product of $\mathrm{k}$ independent density functions, therefore the marginal posterior density functions of $\lambda_{1}, \lambda_{2}, \ldots, \lambda_{k}$, given the data, are $G\left(M_{r}(h)+a_{h}, U_{h}+b_{h}\right)$. To obtain the Bayesian estimation of $\lambda_{1}, \lambda_{2}, \ldots, \lambda_{k}$ we consider three loss functions. The first one, is the squared error (SE) loss function which is symmetric loss function. The second is the LINEX loss function introduced by Varian (1975) which is asymmetric loss function. The LINEX loss function with parameters $\tau$ and $v$ is given by

$$
\ell(\tilde{\theta}, \theta)=v\left\{e^{\tau(\widetilde{\theta}-\theta)}-\tau(\tilde{\theta}-\theta)-1\right\}
$$

where $\tau$ and $v$ are constants. From (6) the Bayes estimator $\tilde{\theta}$ of $\theta$ is given by

$$
\tilde{\theta}_{B L}=-\frac{1}{\tau} \ln E\left(e^{-\tau \theta}\right), \tau \neq 0
$$

The third is the GE asymmetric loss function $\hat{\theta}_{B E}$ proposed by Calabria and Pulcini (1994) and given by

$$
L\left(\tilde{\theta}^{*}, \theta\right) \propto\left(\frac{\widetilde{\theta}^{*}}{\theta}\right)^{c}-c \ln \left(\frac{\widetilde{\theta}^{*}}{\theta}\right)-1,
$$

where $\mathrm{c}$ is a shape parameter whose minimum occurs at $\left(\tilde{\theta}^{*}=\theta\right)$. The Bayes estimate $\tilde{\theta}^{*}$ of $\theta$ under GE loss function in (8) is given by

$$
\tilde{\theta}^{*}=\left(E_{\theta}\left[\theta^{-c}\right]\right)^{\frac{-1}{c}} .
$$

Under SE loss function, the Bayes estimators of $\lambda_{1}, \lambda_{2}, \ldots, \lambda_{k}$ is the posterior mean which can be obtained from (5) as 


$$
\hat{\lambda}_{h B S}=\frac{M_{r}(h)+a_{h}}{U_{h}+b_{h}}, 1 \leq \square \leq k .
$$

It is to be noted that when $a_{h}=b_{h}=0$, for $1 \leq h \leq k$, the Bayes estimators in (10) coincide with the corresponding MLEs in (3). Let $Q_{h}=2 \lambda_{h}\left(U_{h}+b_{h}\right)$ evidently, the pivots $Q_{h}$ follow $\chi_{2\left(M_{r}(h)+a_{h}\right)}^{2}$ distributions, provided that $2 \lambda_{h}\left(M_{r}(h)+a_{h}\right)$ is positive integers. In this case, the $100(1-\alpha) \%$ Bayes credible intervals for $\lambda_{1}, \lambda_{2}, \ldots, \lambda_{k}$ under JPC-I scheme are

$$
\left(\frac{\chi_{2\left(M_{r}(h)+a_{h}\right), 1-\frac{\alpha}{2}}^{2}}{2\left(U_{h}+b_{h}\right)}, \frac{\chi_{2\left(M_{r}(h)+a_{h}\right), \frac{\alpha}{2}}^{2}}{2\left(U_{h}+b_{h}\right)}\right), \square=1,2, \ldots, k
$$

where $\chi_{v, \alpha}^{2}$ is the $\alpha$ percentage point of the $\chi_{v}^{2}$ distribution with $v$ degrees of freedom.

Under LINEX loss function in (6), the Bayes estimators of $\lambda_{1}, \lambda_{2}, \ldots, \lambda_{k}$ are given by

$$
\hat{\lambda}_{h B L}=\frac{M_{r}(h)+a_{h}}{\tau} \ln \left(1+\frac{\tau}{U_{h}+b_{h}}\right), \square=1,2, \ldots, k
$$

where $\tau \neq 0$. Under the GE loss function in (9), the Bayes estimators of $\lambda_{1}, \lambda_{2}, \ldots, \lambda_{k}$ are given by

$$
\hat{\lambda}_{h B E}=\left(\frac{\Gamma\left(M_{r}(h)+a_{h}-c\right)}{\Gamma\left(M_{r}(h)+a_{h}\right)}\right)^{-\frac{1}{c}} \frac{1}{U_{h}+b_{h}}, \square=1,2, \ldots, k .
$$

\section{Numerical Illustration}

This section is devoted to illustrate the theoretical result obtained in the previous sections numerically by analyzing a simulated and real data sets.

\section{Example (1): Real data-set}

To illustrate the usefulness of the proposed estimators obtained in sections 3, 4 and 5 with real situations, we consider Nelson's data (1982, Ch. 10, Table 4.1) which correspond to breakdown in minutes of an insulating fluid subjected to high voltage stress. These failure times were observed in the form of groups with each group reporting data on 10 insulating fluids. Let us consider the following three groups of samples of failure time data presented in Table (1).

Table 1: The failure time data as three groups of insulating fluids

\begin{tabular}{ccccccccccc}
\hline Group & \multicolumn{10}{c}{ Data } \\
\hline 1 & 0.31 & 0.66 & 1.54 & 1.70 & 1.82 & 1.89 & 2.17 & 2.24 & 4.03 & 9.99 \\
2 & 0.00 & 0.18 & 0.55 & 0.66 & 0.71 & 1.30 & 1.63 & 2.17 & 2.75 & 10.60 \\
3 & 0.49 & 0.64 & 0.82 & 0.93 & 1.08 & 1.99 & 2.06 & 2.15 & 2.57 & 4.75 \\
\hline
\end{tabular}

Table (2) presents the JPC-I sample data that have been obtained from the three samples in Table (1) with $T_{1}=1, T_{2}=2$ and $T_{3}=3$ (in minutes). The generated JPC-I sample size is 23 and presented in Table (2) along with the realized values of other pertinent variables. We obtain the MLEs and Bayes estimates of $\lambda_{1}, \lambda_{2}$ and $\lambda_{3}$ (with the choice of $\left(a_{1}, a_{2}, a_{3}, b_{1}, b_{2}, b_{3}\right)=(1.1,1.4,1.6,1,1,1)$ as hyper-parameters values, these results are presented in Table (3). Table (4) shows the asymptotic variance covariance matrix of the MLEs. Table (5) presents the 95\% approximate, Boot-p, Boot-t (with $\quad B=1000$ bootstrap samples) and Bayes credible intervals for $\lambda_{1}, \lambda_{2}$ and $\lambda_{3}$. 
Table 2: The JPC-I sample data from Nelson's data (1982, Ch. 10, Table 4.1)

\begin{tabular}{|c|c|c|c|c|c|c|c|c|c|c|}
\hline \multirow[t]{2}{*}{$j$} & \multirow[t]{2}{*}{$N_{j}$} & \multirow{2}{*}{$\begin{array}{c}\text { Failure } \\
\text { Times } \\
w_{i}\end{array}$} & \multicolumn{3}{|c|}{$z_{i}(\square)$} & \multicolumn{3}{|c|}{$s_{j}(\square)$} & \multirow[t]{2}{*}{$R_{j}$} & \multirow[t]{2}{*}{$r_{i}$} \\
\hline & & & $z_{i}(1)$ & $z_{i}(2)$ & $z_{i}(3)$ & $s_{j}(1)$ & $s_{j}(2)$ & $\overline{s_{j}(3)}$ & & \\
\hline \multirow[t]{11}{*}{1} & 30 & 0.00 & 0 & 1 & 0 & & & & & \\
\hline & & 0.18 & 0 & 1 & 0 & & & & & \\
\hline & & 0.31 & 1 & 0 & 0 & & & & & \\
\hline & & 0.49 & 0 & 0 & 1 & & & & & \\
\hline & & 0.55 & 0 & 1 & 0 & 1 & 0 & 1 & 2 & 11 \\
\hline & & 0.64 & 0 & 0 & 1 & & & & & \\
\hline & & 0.66 & 1 & 0 & 0 & & & & & \\
\hline & & 0.66 & 0 & 1 & 0 & & & & & \\
\hline & & 0.71 & 0 & 1 & 0 & & & & & \\
\hline & & 0.82 & 0 & 0 & 1 & & & & & \\
\hline & & 0.93 & 0 & 0 & 1 & & & & & \\
\hline \multirow[t]{8}{*}{2} & 17 & 1.08 & 0 & 0 & 1 & & & & & \\
\hline & & 1.30 & 0 & 1 & 0 & & & & & \\
\hline & & 1.54 & 1 & 0 & 0 & & & & & \\
\hline & & 1.63 & 0 & 1 & 0 & 0 & 1 & 2 & 3 & 8 \\
\hline & & 1.70 & 1 & 0 & 0 & & & & & \\
\hline & & 1.82 & 1 & 0 & 0 & & & & & \\
\hline & & 1.89 & 1 & 0 & 0 & & & & & \\
\hline & & 1.99 & 0 & 0 & 1 & & & & & \\
\hline \multirow[t]{4}{*}{3} & 6 & 2.06 & 0 & 0 & 1 & & & & & \\
\hline & & 2.17 & 1 & 0 & 0 & 1 & 1 & 0 & 2 & 4 \\
\hline & & 2.24 & 1 & 0 & 0 & & & & & \\
\hline & & 2.75 & 0 & 1 & 0 & & & & & \\
\hline \multicolumn{2}{|c|}{ Tot: } & & & & & 2 & 2 & 3 & 7 & 23 \\
\hline
\end{tabular}

Table 3: The MLE and Bayesian estimates of $\lambda_{1}, \lambda_{2}$ and $\lambda_{3}$

\begin{tabular}{lcccccccc}
\hline & MLEs & \multicolumn{6}{c}{ Bayesian estimates } \\
\cline { 3 - 9 } & & $\begin{array}{c}\text { Squared } \\
\text { error }\end{array}$ & \multicolumn{3}{c}{ LINEX } & \multicolumn{4}{c}{ General entropy } \\
\cline { 3 - 9 } & & & $\tau=0.1$ & $\tau=0.5$ & $\tau=1$ & $c=0.5$ & $c=0.1$ & $c=0.5$ \\
$\hat{\lambda}_{1}$ & 0.49 & 0.525 & 0.524 & 0.518 & 0.511 & 0.511 & 0.494 & 0.482 \\
$\hat{\lambda}_{2}$ & 0.626 & 0.682 & 0.68 & 0.67 & 0.659 & 0.664 & 0.643 & 0.628 \\
$\hat{\lambda}_{3}$ & 0.538 & 0.614 & 0.612 & 0.603 & 0.593 & 0.596 & 0.575 & 0.561 \\
\hline
\end{tabular}

Table 4: Estimates of the asymptotic variance covariance matrix of the MLEs based on JPC-I sample.

Asymptotic variance covariance matrix 


$\left(\begin{array}{ccc}0.03 & 0 & 0 \\ 0 & 0.049 & 0 \\ 0 & 0 & 0.0414\end{array}\right)$

Table 5: The 95\% approximate, Boot-p, Boot-t and Bayes credible intervals for $\lambda_{1}, \lambda_{2}$ and $\lambda_{3}$.

\begin{tabular}{lccc}
\hline Method & $\lambda_{1}$ & $\lambda_{2}$ & $\lambda_{3}$ \\
\cline { 2 - 4 } Approximate & $(0.15,0.829)$ & $(0.192,1.06)$ & $(0.139,0.937)$ \\
Boot-p & $(0.398,0.684)$ & $(0.436,0.974)$ & $(0.33,0.731)$ \\
Boot-t & $(0.394,0.649)$ & $(0.377,0.89)$ & $(0.309,0.781)$ \\
Bayes credible & $(0.241,0.917)$ & $(0.318,1.182)$ & $(0.275,1.087)$ \\
\hline
\end{tabular}

From the results in Tables 3 and 4, we can observe that the estimates to be quite stable. From Tables 5, we also observed that the Boot-t intervals for $\lambda_{1}$ and $\lambda_{2}$ perform better than those based on approximate, Boot-p and Bayes credible intervals. We also observed that the Boot-p interval for $\lambda_{3}$ performing better than other methods.

\section{Example (2) Simulated example}

To illustrate the use of the estimation method proposed in this article, a JPC-I sample are generated from three exponential populations with parameters $\left(\lambda_{1}=2, \lambda_{2}=3\right.$ and $\left.\lambda_{3}=5\right)$, $n_{\square}=15$ and we consider the following schemes:

Scheme (1): $\delta=3,\left(T_{1}=0.1, T_{2}=0.2, T_{3}=0.4\right) \operatorname{and}\left(R_{1}=7, R_{2}=7, R_{3}=2\right)$

Scheme (2): $\delta=5,\left(T_{1}=0.1, T_{2}=0.15, T_{3}=0.2, T_{4}=0.35, T_{5}=0.6\right)$ and

$$
\left(R_{1}=6, R_{2}=5, R_{3}=5, R_{4}=2, R_{5}=1\right)
$$

The JPC-I sample of size 29 from scheme (1) and 26 from scheme (2) are generated and presented in Tables (6) and (7) along with the realized values of other pertinent variables. We compute the MLEs, mean squared errors (MSE) and Bayesian estimates (with the choice of $\left(\left(a_{1}, a_{2}, a_{3}, b_{1}, b_{2}, b_{3}\right)=(1.5,1.5,6,1.1,1.2,1)\right.$. These results are displayed in Table (8). Table (9) presents the $95 \%$ approximate, Boot-p, Boot-t (with $\mathrm{B}=1000$ bootstrap samples) and Bayes credible intervals for $\lambda_{1}, \lambda_{2}$ and $\lambda_{3}$. 
Table 6: The JPC-I censored data from scheme (1)

\begin{tabular}{|c|c|c|c|c|c|c|c|c|c|c|}
\hline \multirow[t]{2}{*}{$j$} & \multirow[t]{2}{*}{$N_{j}$} & \multirow{2}{*}{$\begin{array}{c}\text { Failure } \\
\text { Times } \\
w_{i}\end{array}$} & \multicolumn{3}{|c|}{$z_{i}(\square)$} & \multicolumn{3}{|c|}{$s_{j}(\square)$} & \multirow[t]{2}{*}{$R_{j}$} & \multirow[t]{2}{*}{$r_{i}$} \\
\hline & & & $z_{i}(1)$ & $\overline{z_{i}(2)}$ & $z_{i}(3)$ & $s_{j}(1)$ & $s_{j}(2)$ & $\overline{s_{j}(3)}$ & & \\
\hline \multirow[t]{7}{*}{1} & 45 & 0.009 & 0 & 1 & 0 & & & & & \\
\hline & & 0.009 & 0 & 0 & 1 & & & & & \\
\hline & & 0.010 & 1 & 0 & 0 & & & & & \\
\hline & & 0.067 & 1 & 0 & 0 & 4 & 1 & 2 & 7 & 7 \\
\hline & & 0.073 & 1 & 0 & 0 & & & & & \\
\hline & & 0.095 & 0 & 0 & 1 & & & & & \\
\hline & & 0.097 & 0 & 0 & 1 & & & & & \\
\hline \multirow[t]{12}{*}{2} & 31 & 0.102 & 0 & 1 & 0 & & & & & \\
\hline & & 0.103 & 0 & 1 & 0 & & & & & \\
\hline & & 0.105 & 0 & 0 & 1 & & & & & \\
\hline & & 0.113 & 1 & 0 & 0 & & & & & \\
\hline & & 0.113 & 0 & 0 & 1 & & & & & \\
\hline & & 0.126 & 1 & 0 & 0 & & & & & \\
\hline & & 0.138 & 0 & 0 & 1 & 3 & 2 & 2 & 7 & 12 \\
\hline & & 0.144 & 0 & 1 & 0 & & & & & \\
\hline & & 0.164 & 0 & 0 & 1 & & & & & \\
\hline & & 0.172 & 0 & 1 & 0 & & & & & \\
\hline & & 0.175 & 0 & 0 & 1 & & & & & \\
\hline & & 0.184 & 0 & 0 & 1 & & & & & \\
\hline \multirow[t]{10}{*}{3} & 12 & 0.205 & 0 & 1 & 0 & & & & & \\
\hline & & 0.216 & 0 & 1 & 0 & & & & & \\
\hline & & 0.231 & 0 & 1 & 0 & & & & & \\
\hline & & 0.241 & 0 & 0 & 1 & & & & & \\
\hline & & 0.276 & 1 & 0 & 0 & & & & & \\
\hline & & 0.303 & 0 & 1 & 0 & 2 & 0 & 0 & 2 & 10 \\
\hline & & 0.304 & 0 & 0 & 1 & & & & & \\
\hline & & 0.332 & 0 & 1 & 0 & & & & & \\
\hline & & 0.347 & 0 & 1 & 0 & & & & & \\
\hline & & 0.382 & 0 & 1 & 0 & & & & & \\
\hline \multicolumn{2}{|c|}{ Total } & & & & & 9 & 3 & 4 & 16 & 29 \\
\hline
\end{tabular}


Table 7: The JPC-I censored data from scheme (2)

\begin{tabular}{|c|c|c|c|c|c|c|c|c|c|c|}
\hline \multirow[t]{2}{*}{$j$} & \multirow[t]{2}{*}{$\overline{N_{j}}$} & \multirow{2}{*}{$\begin{array}{c}\text { Failure } \\
\text { Times } \\
w_{i}\end{array}$} & \multicolumn{3}{|c|}{$\overline{z_{i}(\square)}$} & \multicolumn{3}{|c|}{$\overline{s_{j}(\square)}$} & \multirow[t]{2}{*}{$R_{j}$} & \multirow[t]{2}{*}{$\overline{r_{i}}$} \\
\hline & & & $z_{i}(1)$ & $z_{i}(2)$ & $z_{i}(3)$ & $s_{j}(1)$ & $s_{j}(2)$ & $s_{j}(3)$ & & \\
\hline \multirow[t]{6}{*}{1} & 45 & 0.009 & 0 & 1 & 0 & & & & & \\
\hline & & 0.009 & 0 & 0 & 1 & & & & & \\
\hline & & 0.010 & 1 & 0 & 0 & 1 & 2 & 3 & 6 & 6 \\
\hline & & 0.067 & 1 & 0 & 0 & & & & & \\
\hline & & 0.095 & 0 & 0 & 1 & & & & & \\
\hline & & 0.097 & 0 & 0 & 1 & & & & & \\
\hline \multirow[t]{6}{*}{2} & 33 & 0.102 & 0 & 1 & 0 & & & & & \\
\hline & & 0.103 & 0 & 1 & 0 & & & & & \\
\hline & & 0.105 & 0 & 0 & 1 & 1 & 1 & 3 & 5 & 6 \\
\hline & & 0.113 & 1 & 0 & 0 & & & & & \\
\hline & & 0.113 & 0 & 0 & 1 & & & & & \\
\hline & & 0.144 & 0 & 1 & 0 & & & & & \\
\hline \multirow[t]{5}{*}{3} & 22 & 0.164 & 0 & 0 & 1 & & & & & \\
\hline & & 0.172 & 0 & 1 & 0 & 2 & 3 & 0 & 5 & 5 \\
\hline & & 0.175 & 0 & 0 & 1 & & & & & \\
\hline & & 0.184 & 0 & 0 & 1 & & & & & \\
\hline & & 0.196 & 1 & 0 & 0 & & & & & \\
\hline \multirow[t]{5}{*}{4} & 12 & 0.216 & 0 & 1 & 0 & & & & & \\
\hline & & 0.231 & 0 & 1 & 0 & & & & & \\
\hline & & 0.303 & 1 & 0 & 0 & 1 & 1 & 0 & 2 & 5 \\
\hline & & 0.304 & 1 & 0 & 0 & & & & & \\
\hline & & 0.332 & 1 & 0 & 0 & & & & & \\
\hline \multirow[t]{4}{*}{5} & 5 & 0.382 & 0 & 1 & 0 & & & & & \\
\hline & & 0.411 & 1 & 0 & 0 & 1 & 0 & 0 & 1 & 4 \\
\hline & & 0.416 & 0 & 0 & 1 & & & & & \\
\hline & & 0.572 & 1 & 0 & 0 & & & & & \\
\hline To & & & & & & 6 & 7 & 6 & 19 & 26 \\
\hline
\end{tabular}


Table 8: The MLEs and Bayes estimates of $\lambda_{1}, \lambda_{2}$ and $\lambda_{3}$ and MSE's in parentheses using different schemes

\begin{tabular}{|c|c|c|c|c|c|c|c|c|}
\hline \multicolumn{9}{|c|}{ Scheme (1) } \\
\hline & \multirow[t]{3}{*}{ MLEs } & \multicolumn{7}{|c|}{ Bayes estimates } \\
\hline & & \multirow{2}{*}{$\begin{array}{c}\text { Squared } \\
\text { error }\end{array}$} & \multicolumn{3}{|c|}{ LINEX } & \multicolumn{3}{|c|}{ General entropy } \\
\hline & & & $\tau=0.1$ & $\tau=0.5$ & $\tau=1$ & $c=-0.5$ & $c=0.1$ & $c=0.5$ \\
\hline$\hat{\lambda}_{1}$ & $\begin{array}{c}2.434 \\
(0.188)\end{array}$ & $\begin{array}{c}2.104 \\
(0.011)\end{array}$ & $\begin{array}{c}2.075 \\
(0.006)\end{array}$ & $\begin{array}{c}1.969 \\
(0.001)\end{array}$ & $\begin{array}{c}1.854 \\
(0.021)\end{array}$ & $\begin{array}{c}2.035 \\
(0.001)\end{array}$ & $\begin{array}{c}1.951 \\
(0.002)\end{array}$ & $\begin{array}{c}1.895 \\
(0.011)\end{array}$ \\
\hline$\hat{\lambda}_{2}$ & $\begin{array}{c}3.94 \\
(0.884)\end{array}$ & $\begin{array}{c}3.179 \\
(0.032)\end{array}$ & $\begin{array}{c}3.143 \\
(0.020)\end{array}$ & $\begin{array}{c}3.006 \\
(3.6 \mathrm{E}-05)\end{array}$ & $\begin{array}{c}2.855 \\
(0.021)\end{array}$ & $\begin{array}{c}3.121 \\
(0.015)\end{array}$ & $\begin{array}{c}3.051 \\
(0.003)\end{array}$ & $\begin{array}{c}3.003 \\
(9 \mathrm{E}-06)\end{array}$ \\
\hline$\hat{\lambda}_{3}$ & $\begin{array}{c}4.944 \\
(0.003)\end{array}$ & $\begin{array}{c}5.271 \\
(0.073)\end{array}$ & $\begin{array}{c}5.191 \\
(0.036)\end{array}$ & $\begin{array}{c}4.901 \\
(0.010)\end{array}$ & $\begin{array}{c}4.591 \\
(0.167)\end{array}$ & $\begin{array}{c}5.194 \\
(0.038)\end{array}$ & $\begin{array}{c}5.102 \\
(0.010)\end{array}$ & $\begin{array}{c}5.039 \\
(0.006)\end{array}$ \\
\hline \multicolumn{9}{|c|}{ Scheme (2) } \\
\hline$\hat{\lambda}_{1}$ & $\begin{array}{c}2.303 \\
(0.092)\end{array}$ & $\begin{array}{c}2.097 \\
(0.009)\end{array}$ & $\begin{array}{c}2.076 \\
(0.006)\end{array}$ & $\begin{array}{c}1.998 \\
(4 \mathrm{E}-06)\end{array}$ & $\begin{array}{c}1.912 \\
(0.008)\end{array}$ & $\begin{array}{c}2.047 \\
(0.002)\end{array}$ & $\begin{array}{c}1.988 \\
(0.0001)\end{array}$ & $\begin{array}{c}1.948 \\
(0.003)\end{array}$ \\
\hline$\hat{\lambda}_{2}$ & $\begin{array}{c}3.009 \\
(8.1 \mathrm{E}-05)\end{array}$ & $\begin{array}{c}2.462 \\
(0.289)\end{array}$ & $\begin{array}{c}2.43 \\
(0.325)\end{array}$ & $\begin{array}{c}2.315 \\
(0.469)\end{array}$ & $\begin{array}{c}2.189 \\
(0.658)\end{array}$ & $\begin{array}{c}2.398 \\
(0.362)\end{array}$ & $\begin{array}{c}2.32 \\
(0.462)\end{array}$ & $\begin{array}{c}2.268 \\
(0.536)\end{array}$ \\
\hline$\hat{\lambda}_{3}$ & $\begin{array}{c}4.269 \\
(0.534)\end{array}$ & $\begin{array}{c}4.826 \\
(0.030)\end{array}$ & $\begin{array}{c}4.75 \\
(0.063)\end{array}$ & $\begin{array}{c}4.475 \\
(0.276)\end{array}$ & $\begin{array}{c}4.184 \\
(0.666)\end{array}$ & $\begin{array}{c}4.746 \\
(0.065)\end{array}$ & $\begin{array}{c}4.65 \\
(0.123)\end{array}$ & $\begin{array}{c}4.586 \\
(0.171)\end{array}$ \\
\hline
\end{tabular}

Table 9: The 95\% approximate, Boot-p, Boot-t and Bayes credible confidence intervals for $\lambda_{1}, \lambda_{2}$ and $\lambda_{3}$.

\begin{tabular}{lccc}
\hline Method & \multicolumn{3}{c}{ Scheme 1 } \\
\cline { 2 - 4 } & $\lambda_{1}$ & $\lambda_{2}$ & $\lambda_{3}$ \\
\hline Approximate & $(0.486,4.382)$ & $(1.711,6.169)$ & $(2.022,7.865)$ \\
Boot- $p$ & $(0.975,3.794)$ & $(2.261,5.294)$ & $(3.631,6.301)$ \\
Boot- $t$ & $(1.031,3.687)$ & $(2.678,5.156)$ & $(3.782,6.335)$ \\
Bayes credible & $(0.878,3.855)$ & $(1.716,5.086)$ & $(3.071,8.057)$ \\
\hline & & Scheme 2 & \\
\hline Approximate & $(0.798,3.808)$ & $(0.924,5.094)$ & $(1.48,7.059)$ \\
Boot- $p$ & $(1.579,3.164)$ & $(2.021,4.521)$ & $(3.12,6.623)$ \\
Boot- $t$ & $(1.599,3.142)$ & $(2.852,5.178)$ & $(3.488,6.6)$ \\
Bayes credible & $(1.027,3.542)$ & $(1.154,4.257)$ & $(2.701,7.558)$ \\
\hline
\end{tabular}

From Table 8 and 9, it is observed that the Bayes estimates perform better than MLEs in terms of minimum MSE in most of the cases. Comparing the two schemes, we can see that the estimates $\lambda_{1}$ and $\lambda_{2}$ under scheme (2) perform better than those based on scheme (1), while the estimate of $\lambda_{3}$ is better in scheme (1) than scheme (2). Also, it is noted that the approximate and Bayes credible confidence intervals are not satisfactory compared to the Boot-p and Boot-t confidence intervals for both two schemes. Finally, it can be seen that the Boot-p and Boot-t intervals for $\lambda_{1}$ and $\lambda_{2}$ perform better than those based on approximate and Bayes credible intervals in scheme (2) than scheme (1), while Boot-p and Boot-t intervals for $\lambda_{3}$ performing better in scheme (1) than scheme (2).

\section{Conclusions}

In this paper, for $\mathrm{k}$ exponential distributions based on the JPC-I scheme the maximum 
likelihood estimators and the Bayes estimators based on squared error, LINEX and general entropy loss functions are investigated. Also, the approximate, Boot-t, Boot-p confidence and Bayes credible intervals are discussed. We apply a set of real data set by Nelson (1982) and a simulated example for illustration purpose. It is observed that the Bayes estimates perform better than the maximum likelihood estimates in terms of minimum MSE. Also, the Boot-p and Boot-t confidence intervals perform better than other methods. We propose as a future work to investigate the estimation problems of the $\mathrm{k}$ Weibull distributions as a very popular life time distribution based on the JPC-I scheme

\section{References}

[1] Abo-Kasem O. E. and Nassar M. N. (2019). Statistical Inference for Two Weibull Populations Based on Joint Progressive Type-I Censored Scheme. Journal of Data Science. 17 (2), 349- 362.

[2] Abo-Kasem O. E., Nassar M. N., Sanku D. and Rasouli A. (2019). Classical and Bayesian Estimation for Two Exponential Populations based on Joint Type-I Progressive Hybrid Censoring Scheme. American Journal of Mathematical and Management Sciences. 38(4), 373 - 385.

[3] Ashour S. K and Abo-Kasem O. E. (2014a). Bayesian and non-Bayesian estimation for two generalized exponential populations under joint type-II censored scheme. Pakistan Journal of Statistics and Operation Research. 10 (1), 57- 72.

[4] Ashour S. K and Abo-Kasem O. E. (2014b). Parameter estimation for two Weibull populations under joint type-II censored scheme . International Journal of Engineering and Applied Sciences. $5(4), 31-36$.

[5] Ashour S. K and Abo-Kasem O. E. (2014c). Parameter estimation for multiple Weibull populations under joint type-II censoring. International Journal of Advanced Statistics and Probability. 2 (2), 102-107.

[6] Ashour S. K and Abo-Kasem O. E. (2017). Statistical inference for two exponential populations under joint progressive type-I censored scheme. Communications in Statistics-Theory and Methods, 46(7), 3479-3488.

[7] Balakrishnan, N. and Rasouli, A. (2008). Exact likelihood inference for two exponential populations under joint type-II censoring. Computational Statistics \& Data Analysis, 52, 2725 2738 .

[8] Balakrishnan N. and Feng S. (2015). Exact likelihood inference for k exponential populations under joint type-II censoring. Communications in Statistics - Simulation and Computation. 44(3), 591-613.

[9] Balakrishnan N., Feng S. and Kin-yat L.(2015). Exact likelihood inference for k exponential populations under joint progressive type-II censoring. Communications in Statistics - Simulation and Computation. 44(3), 902-923.

[10] Calabria, R. and Pulcini, G. (1994). An engineering approach to Bayes estimation for the Weibull distribution. Microelectronic Reliability, 34 (5),789-802.

[11] Cohen, A.C. (1965). Maximum likelihood estimation in the Weibull distribution based on complete and censored samples. Technometrics, 7, 579-588.

[12] Doostparast, M., Ahmadi, M., Vali and Ahmadi, J. (2013). Bayes estimation based on joint progressive type-II censored data under LINEX loss function. Communications in Statistics - 
Simulation and Computation. 42(8), 1865-1886.

[13] Efron, B. (1982), The Jackknife, the bootstrap and other re-sampling Plans, CBMS-NSF Regional Conference Series in Applied Mathematics, Vol. 38, SIAM, Philadelphia, PA.

[14] Hall, P. (1988), Theoretical comparison of bootstrap confidence intervals, Annals of Statistics, Vol. 16, 927 - 953.

[15] Nelson W. (1982). Applied life data analysis. New York: Wiley.

[16] Parsi, S. Ganjali, M. and Sanjari Farsipour N. (2011). Conditional maximum likelihood and interval estimation for two Weibull populations under joint type-II progressive censoring. Communications in Statistics-Theory and Methods. (40) 2117-2135.

[17] Rasouli, A., and Balakrishnan, N. (2010). Exact likelihood inference for two exponential populations under joint progressive type-II censoring. Communications in Statistics-Theory and Methods. 39 (12), 2172-2191.

[18] Shafay, A. R., Balakrishnan, N. and Abdel-Aty, Y. (2013). Bayesian inference based on a jointly type-II censored sample from two exponential populations. Communications in Statistics Simulation and Computation. 43, 1-14.

[19] Varian, H. R. (1975). A Bayesian approach to real estate assessment, in Studies in Bayesian Econometrics and Statistic in Honor of Leonard J. Savage, S. E. Fienberg and A. Zellner (eds.), North-Holland, Amsterdam, 195-208. 